\title{
Die Positionen der Parteien zur Bundestagswahl 2021: Ergebnisse des Open Expert Surveys
}

\author{
Michael Jankowski (D) Anna-Sophie Kurella • Christian Stecker • \\ Andreas Blätte · Thomas Bräuninger · Marc Debus · Jochen Müller • \\ Susanne Pickel
}

Eingegangen: 29. November 2021 / Überarbeitet: 17. Januar 2022 / Angenommen: 18. Januar 2022 /

Online publiziert: 22. Februar 2022

(C) Der/die Autor(en) 2022

Michael Jankowski $(\bowtie)$

Universität Bremen, Mary-Somerville-Straße 7, 28359 Bremen, Deutschland

E-Mail: jankowsk@uni-bremen.de

Anna-Sophie Kurella · Thomas Bräuninger · Marc Debus

Universität Mannheim, Mannheim, Deutschland

Anna-Sophie Kurella

E-Mail: anna.kurella@mzes.uni-mannheim.de

Thomas Bräuninger

E-Mail: thomas.braeuninger@uni-mannheim.de

Marc Debus

E-Mail: marc.debus@uni-mannheim.de

Christian Stecker

TU Darmstadt, Darmstadt, Deutschland

E-Mail: christian.stecker@tu-darmstadt.de

Andreas Blätte $\cdot$ Susanne Pickel

Universität Duisburg-Essen, Duisburg, Deutschland

Andreas Blätte

E-Mail: andreas.blaette@uni-due.de

Susanne Pickel

E-Mail: susanne.pickel@uni-due.de

Jochen Müller

HU Berlin, Berlin, Deutschland

E-Mail: jochen.mueller@hu-berlin.de 
Zusammenfassung Diese Research Note berichtet zentrale Ergebnisse des Open Expert Surveys 2021 (OES21). In diesem Expert:innen-Survey, der vor der Bundestagswahl 2021 durchgeführt wurde, haben mehr als 300 Politikwissenschaftler:innen die wichtigsten Parteien entlang zentraler politischer Sachfragen verortet und deren Wichtigkeit für die jeweilige Partei geschätzt. Der OES21 unterscheidet sich von gängigen Expert:innenbefragungen in zweierlei Hinsicht. Zum einen umfasst der Survey zahlreiche themenspezifische Items, die in anderen Befragungen bislang keine Beachtung fanden. Zum anderen ist die Anzahl der Expert:innen sehr hoch, wodurch Zusammenhänge in der Positionierung auf Ebene der einzelnen Expert:innen analysiert werden können.

Schlüsselwörter Expertenbefragung · Parteienwettbewerb · Parteipositionen · Bundestagswahl 2021

\section{Party Positions in the German General Election of 2021: Results from the Open Expert Survey}

Abstract In this research note, we present the main findings from the Open Expert Survey 2021 (OES2021). We conducted this expert survey prior to the German General Election of 2021. More than 300 political scientists participated. The experts provided estimates for the relevant German parties on various dimensions and policy issues. They also assessed the salience of specific topics for the parties. Two aspects make the OES21 stand out against other expert surveys. First, it provides party placements on multiple issues, many of which are not asked in existing expert surveys. Second, the number of experts is comparatively large, allowing to analyze in greater detail how expert characteristics affect party placement.

Keywords Expert survey · Party competition · Party positions · German General Election of 2021

\section{Einleitung}

Wenn Politikwissenschaftler:innen Phänomene wie Polarisierung (Dalton 2008), Repräsentationserfolg (Golder und Stramski 2010), Konfliktstrukturen (Albright 2010), Politikergebnisse (Saalfeld 2006) oder Koalitionsbildungsprozesse (Bräuninger et al. 2019b) analysieren, sind Kenntnisse über die inhaltlichen Präferenzen von Parteien und Politiker:innen unverzichtbar. Diese Policy-Präferenzen beeinflussen neben anderen Motiven, wie etwa vote- oder office-seeking (Müller und Strøm 2010) und dem institutionellen Umfeld, die Handlungskalküle politischer Akteure. Die Messung von Parteipositionen gehört daher zum Kerngeschäft der empirischen Parteienforschung (Laver 2014). Dies umfasst in der Regel auch eine Messung davon, welche Dimensionen und Themen von den Parteien als besonders wichtig empfunden werden.

Die Möglichkeiten der Positionsbestimmungen haben sich inzwischen weit entwickelt und ausdifferenziert. Geht es um die Erklärung einzelner konkreter Er- 
eignisse und politischer Prozesse, rekonstruieren Forschende die inhaltlichen Ziele der relevanten Akteure detailliert auf Basis vielfältiger Quellen wie Wahlprogramme, Aussagen von Spitzenpolitiker:innen oder Positionspapiere (z. B. Zohlnhöfer 1999; Ganghof 2006). Für systematische und komparative Perspektiven haben sich verschiedene quantitative Verfahren etabliert, die Positionen auf höher aggregierten Dimensionen schätzen. Ein Zweig nutzt hierfür Partei- und Wahlprogramme. Das Manifesto Project hat z.B. ein manuelles Kodierverfahren etabliert, um aus den verschiedenen Kategorien die Wichtigkeit und Positionen der Parteien abzuleiten (Volkens et al. 2014). Gleichzeitig wurden eine Reihe von (teil-)automatisierten Textanalyseverfahren entwickelt, welche die Positionen von Partien anhand von Wahlprogrammen schätzen (Laver et al. 2003; Slapin und Proksch 2008). Beispielsweise kartieren Bräuninger et al. (2020) den positionalen Parteienwettbewerb in den Bundesländern seit 1990 auf Basis einer Wordscores-Analyse aller Wahlprogramme und Koalitionsverträge. Ähnliche Einsichten liegen inzwischen auch für die kommunale Ebene vor (Gross und Jankowski 2020).

Ein weiterer Zweig gewinnt Positionsdaten über Befragungen. In jüngerer Zeit haben etwa Crowdsourcing-Ansätze an Bedeutung gewonnen, bei welchen die Positionen der Parteien durch die Befragung einer Vielzahl von Wähler:innen geschätzt werden (Benoit et al. 2016; Horn 2019). Etablierter ist jedoch die Befragung von Expert:innen. Zum Grundkanon gehört hier die ländervergleichende Befragung von Benoit und Laver (2006), die einflussreiche Vorläufer fortschreibt (Laver und Hunt 1992). Auch die bis 1999 zurückreichende Zeitreihe des Chapel Hill Expert Survey (Polk et al. 2017) gehört dazu. Die Messung von Präferenzen und die Interpretation der Daten werfen jeweils eigene methodologische und praktische Probleme auf (Ganghof 2003; Laver 2014). Für zahlreiche empirische Fragestellungen der Politikwissenschaft haben sich diese Verfahren jedoch als äußerst fruchtbar erwiesen.

Diese Research Note stellt den Open Expert Survey 2021 (OES21) vor, der in der Tradition von Expert:innenbefragungen steht. Der OES21 knüpft an den Survey von Bräuninger et al. (2019a) zur Bundestagswahl 2017 an und setzt die enthaltenen Zeitreihen zu zahlreichen politischen Dimensionen fort. Der OES21 geht allerdings über frühere Projekte hinaus, indem er weitere von der politikwissenschaftlichen Gemeinschaft nachgefragte Items ins Feld bringt und konsequent in einem open science-Rahmen umgesetzt wird. Sämtliche Daten des OES21 können unter https:// oes21.de/ bzw. direkt über das Harvard Dataverse (https://doi.org/10.7910/DVN/ XIL6OW) heruntergeladen werden.

Im nächsten Abschnitt berichten wir zunächst über die mehrstufige Durchführung des OES21. Anschließend werfen wir verschiedene Schlaglichter auf zentrale Ergebnisse der Befragung. Zunächst berichten wir deskriptiv die Positionen der einzelnen Themenbereiche und gehen auf die Positionsverschiebungen zwischen 2017 und 2021 ein. Weiterhin prüfen wir, ob die Positionierungen davon beeinflusst sind, welche Expert:innen jeweils befragt wurden. Abschließend widmen wir uns der Dimensionalität des Politikraums und schließen mit grundsätzlicheren Überlegungen zur Durchführung und Etablierung von offenen Expert:innen-Befragungen. 


\section{Offene Expert:innenbefragung}

Der OES21 wurde in einem mehrstufigen Prozess konzipiert und durchgeführt. Als Kernmodul waren vorab acht Items eingeplant, die zentrale Politikfelder abdecken und die Zeitreihen früherer Expertenbefragungen fortsetzen (z. B. Laver und Hunt 1992; Benoit und Laver 2006). Die anvisierten Politikfelder umschließen u. a. die Themen Wirtschafts- und Gesellschaftspolitik, Einwanderung, Umwelt, Bildung und Europäische Integration. Die entsprechenden Positionen sollten anhand zeitstabiler und damit vergleichbarer Items erhoben werden.

Wie in früheren Befragungen sollte neben den Positionen auch erhoben werden, welche Wichtigkeit bzw. Salienz die Parteien einzelnen Themenbereichen zuschreiben. Neben den fortzuschreibenden Items vergangener Befragungen enthält der OES21 zahlreiche weitere Items. Dazu haben wir uns im Juni 2021 über verschiedene Email-Verteiler mit einem Call for Items an die politikwissenschaftliche Gemeinschaft gewandt und darum gebeten, weitere Items vorzuschlagen, die für aktuelle Fragen der Parteienforschung von allgemeiner Bedeutung sind und in einer Expert:innenbefragung sinnvoll erhoben werden können. Auf unseren Call meldeten sich 20 Kolleg:innen und benannten mehr als 40 weitere Items. Diese Items erweitern beispielsweise den betrachteten Politikraum hinsichtlich Themen wie Außenpolitik, Corona, Populismus, Terrorismusbekämpfung oder Gleichstellung. Andere vorgeschlagene Items differenzieren bestehende Politikfelder in weitere Subdimensionen aus. Noch andere Items gehen über die inhaltliche Positionierung von Parteien hinaus und fokussieren etwa auf Repräsentationsbeziehungen von Parteien zu sozialen Gruppen, innerparteiliche Nominierungskriterien von Kanzlerkandidat:innen oder Charakterisierungen innerparteilicher Konflikte.

In Korrespondenz mit den Einreichenden wurden der Itemkatalog um Redundanzen bereinigt und Formulierungen optimiert. Im Sinne der Zeitreihenkonsistenz wurden alle vorgeschlagenen Skalen auf die zuvor z. B. in Benoit und Laver (2006) genutzten endpunktbezeichneten Skalen von 1 bis 20 harmonisiert. Diese Skalierung erscheint einigen als übertrieben feinkörnig; sie kann aber problemlos auf eine gröbere Unterscheidung transformiert werden. Gleichzeitig kann kritisch angemerkt werden, dass eine solch feinkörnige Messung auch zu Problemen führen kann, da sie die Positionsbestimmung komplexer macht, als wenn es nur einige wenige Positionen zur Auswahl gäbe. Höhere Abbruchraten oder Non-Response bei einzelnen Items könnten die Folge sein. Daher ist in zukünftigen Vorhaben eventuell eine Reduktion der Antwortkategorien sinnvoll. Auch in diesem Fall ließen sich noch Zeitreihen analysieren, indem man die Antwortskalen früherer Wellen entsprechend zusammenfasst.

Der so konsolidierte Fragenkatalog wurde für einen Pre-Test im Team des OES21 und unter den Einreichenden zirkuliert. Neben der weiteren Optimierung von Fragen ergab der Pre-Test u.a. die Einsicht, dass der Survey mit einer geschätzten Gesamtbearbeitungszeit von 45 Minuten deutlich zu umfangreich geraten und damit eine niedrige Rücklaufquote durch vorzeitige Abbrüche zu befürchten war. Da der OES21 möglichst vielen Kolleg:innen relevante Forschungsdaten zugänglich machen soll, wollten wir jedoch weitere Item-Kürzungen vermeiden. Das Zeitproblem wurde über eine bessere Nutzersteuerung im Surveydesign gelindert. So konnten die 
Respondenten die Themengebiete ${ }^{1}$ auswählen, in denen sie ihre Expertise verorten. Nach den Kernitems wurden ihnen dann nur die entsprechenden Items aus den vorab gewählten Bereichen vorgelegt.

In der vorliegenden Research Note berichten wir die acht Kernitems und zusätzlich die generelle Links-Rechts-Position. Konkret umfassen die Items die folgenden politischen Themenbereiche:

- Links-Rechts ( $1=$ ganz links, $20=$ ganz rechts $)$

- Wirtschaftspolitik ( 1 = für Steuererhöhungen/mehröffentliche Leistungen, 20 = für Steuersenkungen/weniger öffentliche Leistungen)

- Gesellschaftspolitik ( 1 = für eine liberale Politik in Fragen wie Abtreibung, Homosexualität und Sterbehilfe; $20=$ gegen eine liberale Politik in diesen Fragen)

- Recht und Ordnung ( 1 = für den Schutz bürgerlicher Freiheiten, 20 = für eine stärkere Kriminalitätsbekämpfung)

- Klima ( 1 = für Umwelt- und Klimaschutz, auch wenn es zu Lasten des Wirtschaftswachstums geht, 20 = für Wirtschaftswachstum, auch wenn es zu Lasten des Klimaschutzes geht $)^{2}$

- Zuwanderung (1=Zuwanderung erleichtern, 20=Zuwanderung erschweren)

- Integration (1= Assimilation, 20= Multikulturalismus)

- Bildung ( 1 = für Gemeinschaftsschulen, 20 = für mehrgliedriges Schulsystem)

- Europäische Integration (1 = mehr Kompetenzen für Europäische Union, 20 = weniger Kompetenzen für Europäische Union)

Neben den Positionen wurde abgefragt, welche Bedeutung der jeweilige Themenbereich für die einzelnen Parteien besitzt. Die Skala rangiert zwischen 1 (gar nicht wichtig) und 20 (sehr wichtig). Bei allen Fragen konnten die Befragten auch „keine Angabe" auswählen.

Zur Schätzung von Position und Wichtigkeit haben wir alle im 20. Bundestag (2017-2021) vertretenen Parteien eingeschlossen, also CDU, CSU, SPD, FDP, Bündnis 90/Die Grünen, Die Linke und die AfD. Eine Differenzierung zwischen CDU und CSU erschien uns sinnvoll, um programmatische Unterschiede abzubilden, die seit der Flüchtlingskrise von 2015 zwischen beiden Unionsschwestern klar und in unterschiedlichen Themenbereichen (z. B. Einwanderung, „Mütter-Rente“) hervortreten. Wir haben auch die Freien Wähler berücksichtigt, die sich erstmals an einer Bundestagswahl beteiligten und von einigen Beobachtern als relevante Kraft eingeschätzt wurden.

Der Survey wurde in Qualtrics programmiert, einer etablierten Umfragesoftware des gleichnamigen US-amerikanischen Softwareunternehmens. Insgesamt wurden 2479 aktive Politikwissenschaftler:innen über Email kontaktiert und zur Befragung eingeladen. Die entsprechenden Email-Adressen haben wir in einer umfangreichen

\footnotetext{
${ }^{1}$ Konkret handelt es sich um die folgenden fünf Themengebiete: Außen- und Sicherheitspolitik, Bildungspolitik, Europäische Union, Gender- und Familienpolitik sowie die Interessensvertretung von gesellschaftlichen Gruppen.

${ }^{2}$ Die Frage ist an die Formulierung aus dem Chapel Hill Expert Survey angelehnt. Die Formulierung impliziert einen möglichen Gegensatz zwischen Klima-/Umweltschutz und Wirtschaftswachstum, der so sicherlich nicht von allen geteilt wird. Eine Umformulierung des Items in zukünftigen Wellen wäre daher überlegenswert.
} 
Inspektion von Instituts- und Lehrstuhlwebseiten aller politikwissenschaftlichen Institute an deutschen Universitäten zusammengetragen. Verwandte Disziplinen, wie Soziologie oder Wirtschaftswissenschaften wurden nicht eingeschlossen. Erstmalig haben wir am 20. August 2021 zur Teilnahme am OES21 eingeladen, am 10. September 2021 wurde eine Erinnerung verschickt. Am 24. September wurde der OES21 endgültig geschlossen.

\section{Beschreibung der Expert:innen}

Insgesamt liegen die Antworten von 361 Expert:innen vor. Allerdings umfasst diese Zahl auch Expert:innen, welche den OES21 nicht vollständig ausgefüllt haben und z.B. nach der Hälfte abgebrochen haben. Wir haben abgebrochene Surveys nur in den Daten behalten, wenn diese mind. 10\% des Surveys bearbeitet haben und keine offensichtlichen Inkonsistenzen beinhalteten, die auf ein testweises Anklicken des Survey schließen ließen. ${ }^{3}$ Insgesamt haben 192 (53\%) den OES21 vollständig ausgefüllt und 169 (47\%) nicht vollständig. Weiterhin ist zu beachten, dass die Expert:innen keine Positionierungen vornehmen mussten. Bei jedem Item und jeder Partei konnten die Expert:innen bewusst auf Angaben verzichten oder das jeweilige Item einfach frei lassen. Somit schwankt die Anzahl an Antworten auch zwischen den einzelnen Items und Parteien. Ein konsistentes Muster ist dabei, dass die Freien Wähler deutlich seltener positioniert wurden als die anderen Parteien. In der Gesamtschau der erhaltenen Antworten erreicht der OES21, trotz seiner beträchtlichen Länge, höhere Zahlen als z. B. Benoit/Laver (98 Antworten) oder der Chapel-Hill Expert Survey 2019 für die Bundesrepublik Deutschland (bspw. 29 Antworten für die CDU). ${ }^{4}$

\subsection{Teilgebiete und akademischer Grad}

Um einen besseren Überblick über die Expert:innen zu bekommen und auch um zu analysieren, ob die Positionierung der Parteien sich evtl. zwischen den Teildisziplinen unterscheidet, wurden die Expert:innen nach ihren inhaltlichen Forschungsschwerpunkten und akademischen Graden gefragt. Bei den inhaltlichen Forschungsschwerpunkten konnten max. drei Bereiche ausgewählt werden. ${ }^{5}$ Der Karrierefortschritt wird anhand des höchsten akademischen Titels gemessen (nicht promoviert, promoviert, habilitiert oder (Junior-)Professur). Die Verteilung der jeweiligen Nennung ist in Abb. 1 abgebildet. Der Großteil der Expert:innen stammt aus der Gruppe der (noch) nicht Promovierten, gefolgt von der Gruppe der Promovierten ohne Professur oder Habilitation. Diese Verteilung liegt vermutlich daran, dass diese Gruppen rein nummerisch den größten Teil der angeschriebenen Expert:innen ausmachen.

\footnotetext{
3 Bspw. sollte kein:e Expert:in die AfD linker als die Grünen oder Linke einschätzen.

${ }^{4}$ Die Rücklaufquote des OES21 ist verständlicherweise erheblich geringer als bei spezialisierten Expert Surveys, da der OES21 bewusst eine sehr breite Gruppe an Politikwissenschaftler:innen angeschrieben hat.

5 Die durchschnittliche Anzahl an ausgewählten Teilgebieten liegt bei 2,35.
} 


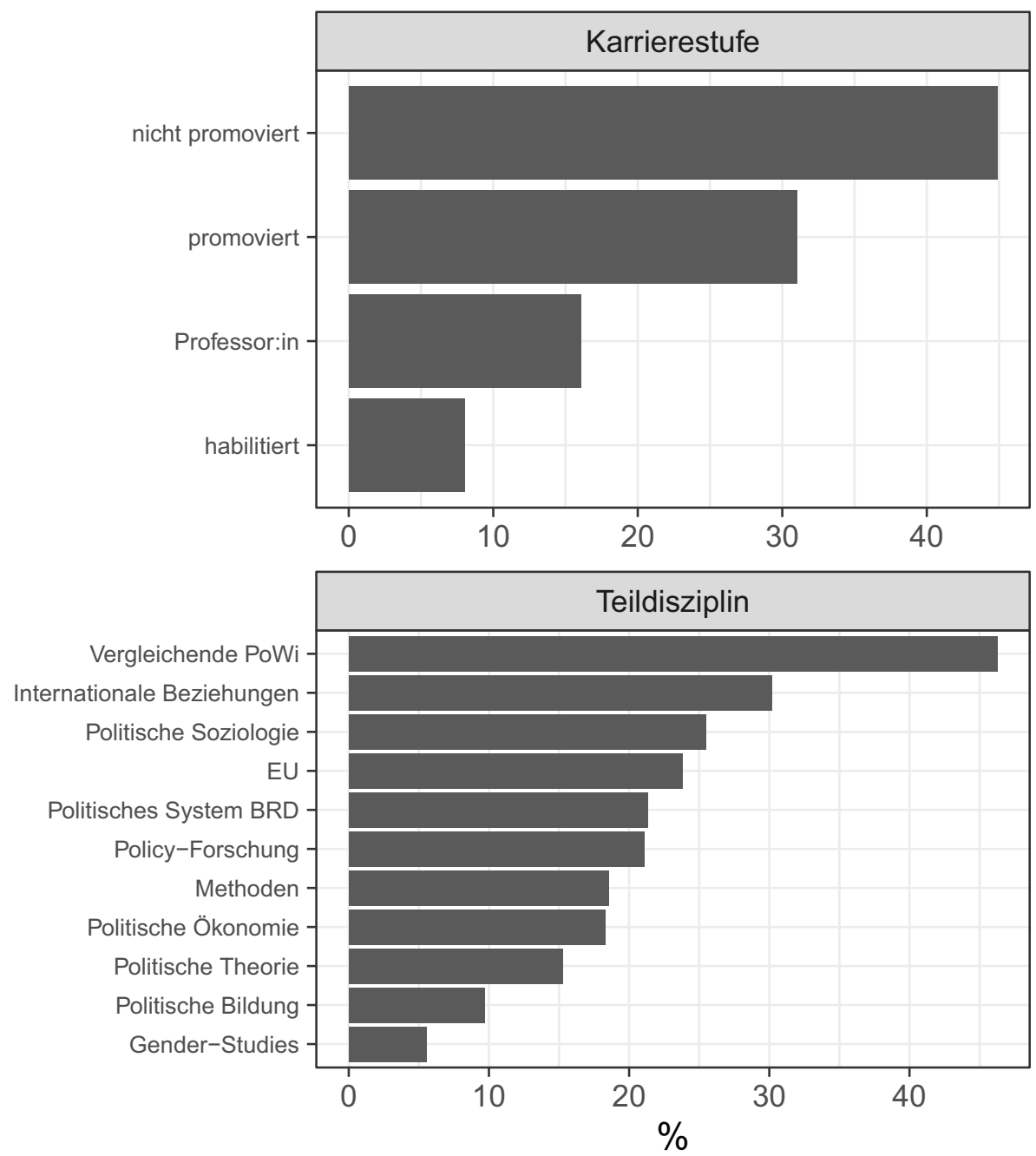

Abb. 1 Zusammensetzung der Expert:innen nach Forschungsschwerpunkt und akademischen Grad

Entsprechend gering ist auch der Anteil an Expert:innen, deren höchster akademischer Grad die Habilitation ist.

Bei der Verteilung der Forschungsschwerpunkte zeigt sich, dass die Vergleichende Politikwissenschaft am häufigsten genannt wird. $46 \%$ aller Expert:innen geben diese Teildisziplin als Schwerpunkt an. Die weiteren Teildisziplinen schwanken in ihren Anteilen zwischen 15 und 30 Prozent und decken unterschiedliche Themen ab. Mit jeweils weniger als 10\% sind „Politische Bildung“ und „Gender Studies“ relativ selten vertreten. 


\subsection{Politische Nähe}

Der OES21 umfasst auch ein Item, welches die Expert:innen nach ihrer Nähe zu den einzelnen Parteien fragt. Diese Informationen sind relevant, um die Effekte der politischen Nähe auf die Positionierung der jeweiligen Parteien einschätzen zu können. Dem Vorgehen des OES21 folgend, wurde die Nähe auch auf einer 20er Skala gemessen, wobei 1 für eine maximale Nähe und 20 für eine maximale Distanz einer Partei zu den eigenen politischen Ansichten steht.

Die Ergebnisse sind in Abb. 2 beschrieben. Auffällig ist hierbei zunächst, dass es sehr wenige Befragten gibt, welche sich der AfD nahe fühlen. Fast alle Befragten nutzen den Endpunkt der Skala, um eine maximale Distanz zur AfD zum Ausdruck zu bringen. Bei den anderen Parteien gibt es eine deutlich größere Spannweite. Insgesamt lässt sich eine größere Nähe der Expert:innen zu Parteien des linken Spektrums, insbesondere zu Grünen und SPD feststellen. Parteien aus dem liberalkonservativen Lager werden in der Regel als deutlich weiter entfernt von den eigenen Präferenzen wahrgenommen, aber es ist gleichzeitig auch nicht so, dass es keine Expert:innen gibt, die sich diesen Parteien nahe fühlen. Analysiert man die Nähe zu den Parteien in Abhängigkeit vom akademischen Status, zeigen sich kleinere Unterschiede zwischen den Gruppen. So zeigen (Junior-)Professor:innen und Habilitierte eine deutlich größere Distanz zur Linken (Mittelwert von 11,2 bzw. 12,9) als die Gruppe der Promovierten $(9,0)$ bzw. nicht Promovierten $(9,5)$. Bei den anderen eher linken Parteien, SPD und Bündnis 90/Die Grünen, zeigen sich solche Unterschiede zwischen den Gruppen jedoch nicht. Auch zeigt sich nur eine leichte Tendenz, dass Parteien aus dem eher rechten Lager durch Professor:innen der eigenen Position als näher beschrieben werden. So liegt die durchschnittliche Nähe der Professor:innen zur FDP bei etwa 12, während sie in den anderen Gruppen bei 13 (nicht Promovier-

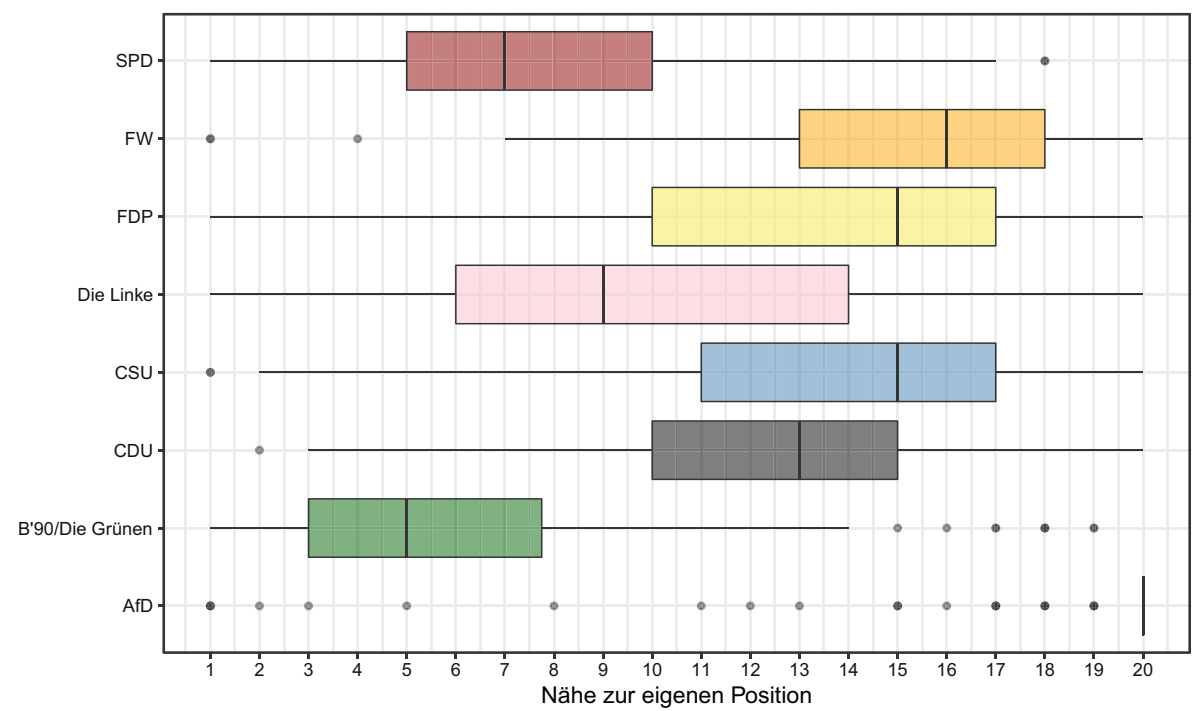

Abb. 2 Nähe der Expert:innen zu den jeweiligen Parteien 
te und Habilitierte) bzw. 14 (Promovierte) liegt. Bei der AfD geben die befragten Professor:innen die größte durchschnittliche Distanz an (19,3 im Vergleich zu unter 19 bei allen anderen Gruppen.)

\section{Positionierung der Parteien auf einzelnen Politikdimensionen}

Der OES21 umfasst insgesamt über 70 Items. Neben der allgemeinen Links-RechtsSkala erfassen 22 Items die Parteipositionen zu sachpolitischen Themen, die somit als klassische Positionsissues betrachtet werden können. Hier soll zunächst auf die Positionierung der Parteien auf der allgemeinen Links-Rechts-Skala sowie auf den acht oben genannten Kernthemen eingegangen werden. Abb. 3 zeigt die Parteiposition als Mittelwert der Expert:inneneinschätzungen und den zugehörigen Standardfehler. Insgesamt nehmen die Befragten deutliche Unterschiede zwischen den Parteien wahr. Je nach Politikfeld besetzen unterschiedliche Parteien die jeweiligen Pole. Während die Parteikonfiguration auf der allgemeinen Links-Rechts-Skala sehr
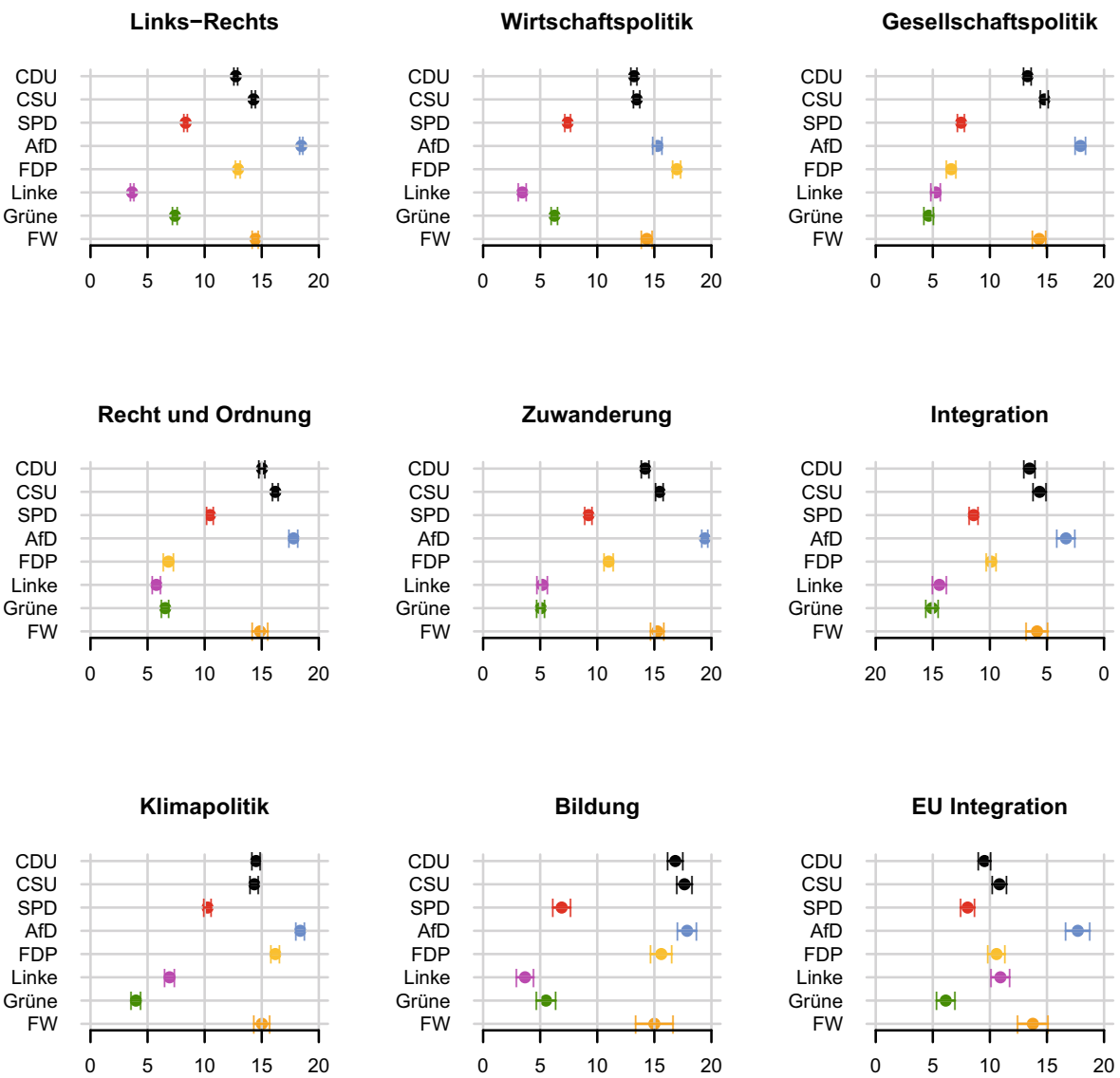

Abb. 3 Mittlere Parteipositionen und Standardfehler auf einzelnen Politikdimensionen 
differenziert ist, erkennt man auf anderen Dimensionen eine stärkere Polarisierung zwischen einzelnen Parteien und/oder politischen Lagern.

Wie zu erwarten, markiert die Linke auf der Links-Rechts-Skala den linken und die AfD den rechten Extrempunkt. Dazwischen ordnen sich die Grünen und die SPD links des Skalenmittelwertes, die CDU, die CSU und die FDP rechts des Mittelwertes ein. Auffällig ist hier die deutliche Diskrepanz zwischen der CDU und der bayerischen Schwesterpartei CSU, welche rechts der CDU eingeschätzt wird. Die Freien Wähler werden in etwa auf der Position der CSU eingeschätzt.

In der Wirtschaftspolitik sehen wir eine Polarisierung zwischen einem linken Lager bestehend aus Linken, Grünen und SPD, und einem rechten Lager bestehend aus CDU, CSU, Freien Wählern, AfD und FDP. Hier wird kaum ein Unterschied zwischen CDU und CSU wahrgenommen. Die FDP tritt als wirtschaftsliberalste Polpartei auf. Dieses Konstellation spiegelt die Positionen zu den prominenten wirtschaftspolitischen Themen der Steuern und Schulden wider. Während die Parteien des linken Lagers in ihren Bundestagswahlprogrammen allesamt Steuererhöhungen für Unternehmen und Spitzenverdienende und eine Beibehaltung des Solidaritätszuschlags forderten, lehnten die Parteien des rechten Lagers Steuererhöhungen kategorisch ab, forderten die Streichung des Solidaritätszuschlags und proklamierten ein Festhalten an der Schuldenbremse.

Auch in der Gesellschaftspolitik erkennt man eine Polarisierung zwischen zwei Lagern. Allerdings nimmt die FDP hier eine liberale Position ein und wird zwischen den Grünen, der Linken und der SPD eingestuft. Wie auch aus den öffentlichen Verlautbarungen und Programmen bekannt, vertreten diese vier Parteien liberale Standpunkte zu Themen wie Abtreibung, Sterbehilfe und Homosexualität. CDU, CSU und die Freien Wähler nehmen zu diesen Fragen eine konservative Position ein, die AfD bildet hier den Extrempunkt. Dies entspricht dem bekannten Muster im gesellschaftspolitischen Konfliktraum, das als Benelux-Konstellation bekannt ist (Laver und Hunt 1992). Abweichungen ergeben sich jedoch auf dem Politikfeld Recht und Ordnung. Hier wird die Position der SPD als weniger liberal wahrgenommen als auf der gesellschaftspolitischen Dimension. Genau genommen wird die SPD knapp rechts des Skalenmittelwertes verordnet und gilt somit als Befürworterin einer stärkeren Kriminalitätsbekämpfung. Außerdem nehmen sowohl CDU als auch CSU einen autoritäreren Standpunkt ein, und werden näher an der rechten AfD verortet, welche auch hier den Extrempol markiert.

Betrachtet man die Parteienkonstellation zu den Themenbereichen der Zuwanderung und der Integration, so erkennt man ein sehr ähnliches Muster wie im Bereich Recht und Ordnung. Lediglich die FDP weicht deutlich ab, indem sie eine weniger liberale Position einnimmt. Zur Frage, ob Zuwanderung erleichtert oder erschwert werden sollte, wird die Position der FDP leicht rechts des Skalenmittelwerts eingeschätzt. Beim Thema Integration bilden Assimilation und Multikulturalismus die Gegensätze. Hier wird die FDP wiederum genau auf dem Mittelpunkt eingeordnet.

Für die Klimapolitik definierte das entsprechende Item einen Konflikt zwischen Klima- und Umweltschutz einerseits, und Wirtschaftswachstum andererseits (Benoit und Laver 2006). Hier nehmen die Grünen erwartungsgemäß den linken Pol ein und werden als stärkste Vertreterin von Klimaschutz und umweltfreundlicher Politk gesehen. Die Linke wird ebenfalls im klimafreundlichen Skalenbereich ver- 
ortet, während die SPD am Mittelpunkt eingestuft wird. Den wirtschaftsfreundlichen Gegenpol nehmen die AfD und die FDP ein.

Im Bereich der Bildungspolitik wird nach der Parteiposition im Konflikt zwischen der Einführung von Gemeinschaftsschulen und dem Festhalten am dreigliedrigen Schulsystem gefragt. Hier ergibt sich dieselbe Polarisierung der Parteigruppen wie auf der wirtschaftspolitischen Konfliktdimension mit der Linken, den Grünen und der SPD als Befürworterinnen von Gemeinschaftsschulen und allen anderen Parteien als Befürworterinnen des dreigliedrigen Schulsystems. Jedoch ist darauf hinzuweisen, dass die Entscheidung über diese Streitfrage außerhalb des Kompetenzbereichs der Bundesebene liegt, und das Thema im Bundestagswahlkampf auch keine besondere Rolle spielte.

In der Frage nach einer Erweiterung der EU-Kompetenzen sehen wir eine deutliche Diskrepanz zwischen der rechten Position der AfD, welche für einen Abbau der Kompetenzen der Europäischen Union steht, und den moderaten Positionen aller anderen Parteien. Die Grünen nehmen den linken Pol ein.

\section{Vergleich der Parteipositionen 2017 und 2021}

Haben die Parteien ihre Positionen im Vergleich zur letzten Bundestagswahl verändert? Wenn ja, in welche Richtung? Ein Vergleich mit den Ergebnissen des Expertensurveys 2017, der identische Items nutzte, gibt Aufschluss. Wir betrachten die von den Expert:innen wahrgenommenen Parteipositionen in den Politikfeldern Wirtschaftspolitik, Gesellschaftspolitik, Klimapolitik, Zuwanderung und auf der allgemeinen Links-Rechts-Skala. Auf der allgemeinen Links-Rechts-Skala erkennen wir lediglich marginale Positionsänderungen, was für eine grundlegende Stabilität der Parteienkonfiguration spricht. In den spezifischen Politikfeldern erkennen wir dagegen größere Sprünge einzelner Parteien, sowie einen grundlegenden Trend zur Depolarisierung.

Abb. 4 veranschaulicht die Bewegungen der Parteien von 2017 zu 2021. Im Bereich der Wirtschaftspolitik sehen wir einen allgemeinen Trend zu wirtschaftsliberaleren Positionen aller Parteien, mit Ausnahme der Grünen, welche ihre Position beibehalten, und der FDP, welche leicht nach links rückt. Somit verkleinert sich der Abstand zwischen der linken und rechten Polpartei in der Wirtschaftspolitik. Ein ähnliches Muster ist im Bereich der Gesellschaftspolitik zu beobachten. Auch hier bewegen sich die Polparteien Grüne und AfD in der Wahrnehmung der Expert:innen aufeinander zu. Doch auch die übrigen Parteien tragen zu einer Abnahme der Polarisierung bei, indem sie sich auf den Skalenmittelpunkt zubewegen: während SPD, FDP und zum kleinen Teil auch die Linke nach rechts rücken, bewegt sich die CSU nach links.

In den Bereichen Klima und Zuwanderung beobachten wir andere Muster. Die Grünen nehmen in beiden Bereichen moderatere Positionen ein als 2017, während die Linke weiter nach links rückt. SPD und CDU unternehmen keine großen Positionsänderungen. Die CSU wird in der Zuwanderungspolitik 2021 jedoch näher an der CDU verortet als 2017. Die FDP nimmt in beiden Bereichen moderatere Positionen ein. Die AfD rückt in der Klimapolitik weiter nach rechts und löst so- 
Wirtschaftspolitik

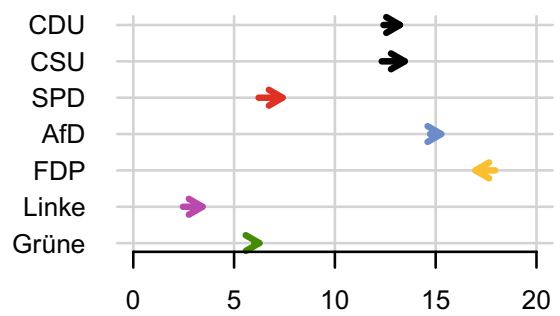

Klimapolitik

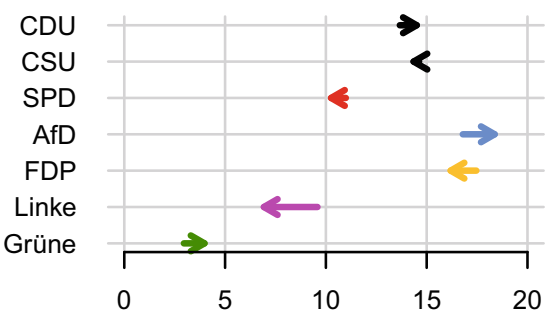

Gesellschaftspolitik

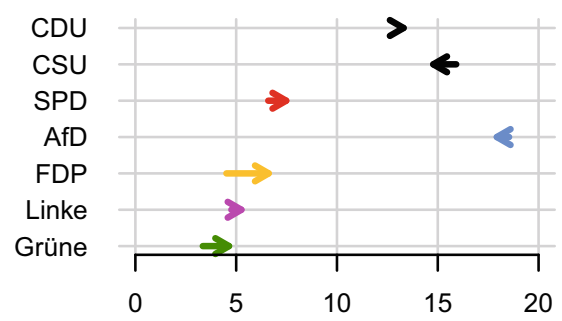

Zuwanderung

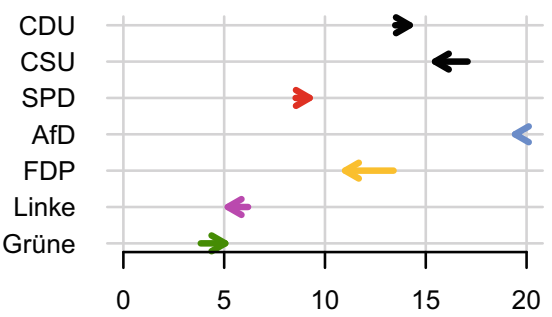

\section{Links-Rechts}

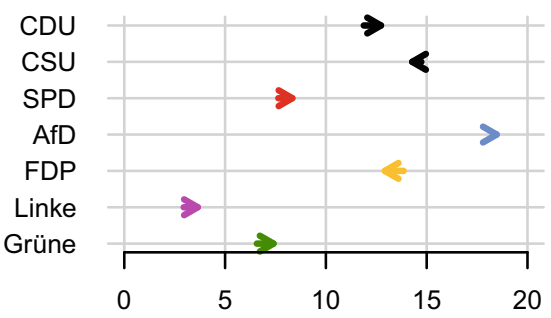

Abb. 4 Veränderung der mittleren Parteipositionen von 2017 zu 2021

mit die FDP als Verteterin der wirtschaftsfreundlichsten Politik im Zielkonflikt mit stärkerem Umweltschutz ab.

\section{Unterschiede in der Positionierung nach Expert:innen-Gruppen}

Der OES21 zeichnet sich durch eine vergleichsweise hohe Anzahl an befragten Expert:innen aus, was den Standardfehler des Mittelwertes verringert. Die hohe Fallzahl versetzt uns zudem in die Lage, systematische Einflüsse auf die Positionsschätzungen freizulegen. Konkret untersuchen wir, inwiefern Eigenschaften der Expert:innen auf ihre Schätzungen wirken. Die resultierenden Einblicke können z. B. dabei helfen, die Werte anderer Erhebungen einzuordnen. Beispielsweise befragt 
der etablierte Chapel Hill Expert Survey nur eine vergleichsweise geringe Anzahl von Personen, die allesamt mindestens promoviert sind und direkt zur Parteipolitik forschen. Beim OES21 ist die Expert:innen-Basis bewusst diverser und umfasst Politikwissenschaftler:innen aller Statusgruppen und Teilgebiete (siehe oben).

Ob unterschiedliche Gruppen von Expert:innen Parteipositionen systematisch unterschiedlich wahrnehmen, betrachten wir im Folgenden anhand der allgemeinen Links-Rechts-Skala. Die Positionierungen der Parteien auf dieser Skala bilden die abhängige Variable in einer Regression, bei welcher wir das Forschungsteilgebiet und den akademischen Status als unabhängige Variable nutzen und auch berücksichtigen, ob ein:e Expert:in den Survey abgeschlossen hat. Beachtet werden sollte, dass jede:r Expert:in max. drei Teilgebiete als Forschungsschwerpunkte angeben konnte. Die Teilgebiete sind daher nicht gegenseitig ausschließend. Die im Modell inkludierten Dummy-Variablen geben somit jeweils den Effekt an, dass eine Person zu dem jeweiligen Teilgebiet forscht, im Vergleich zu einer Person, die nicht in dem Teilgebiet forscht. Beim akademischen Status hingegen unterscheiden wir zwischen Promovierenden, Promovierten, Habilitierten und Professor:innen (einschließlich Junior-Professor:innen). Die Promovierenden sind die Referenzgruppe in der Analyse. Um zu überprüfen, ob der vorzeitige Abbruch der Umfrage durch die Expert:innen einen Effekt auf deren Positionierungen hat, haben wir auch hierfür einen Dummy eingefügt.

Die Ergebnisse sind in Abb. 5 dargestellt. Sie zeigen, dass in den meisten Fällen kein signifikanter Effekt von Forschungsgebiet und akademischem Status auf die Positionierung der jeweiligen Partei ausgeht. Einigkeit besteht insbesondere bei AfD, Linken und FDP. Bei Grünen, CDU, CSU und SPD neigen Personen mit Habilitation oder Professur dazu, diese Parteien weiter links einzuordnen als Promovierende. Allerdings liegen die beobachteten Unterschiede bei maximal einem Skalenpunkt - sie sind angesichts der verwendeten 20er-Skala also sehr gering. Die marginalen Unterschiede können verschiedene Ursachen haben. Eine mögliche Erklärung wäre, dass jüngere Expert:innen andere politische Präferenzen haben als eher ältere Professor:innen und sich dies in einer distinkten Wahrnehmung des politischen Raums niederschlägt. Leichte Hinweise darauf haben wir weiter oben bereits diskutiert. Um dies zu überprüfen, haben wir den Modellen eine Variable hinzugefügt, welche die politische Nähe der Expert:innen zu der jeweiligen Partei inkludiert. Die Ergebnisse zeigen, dass unter Einschluss dieser Variable tatsächlich kaum noch signifikante Unterschiede auftreten.

Kaum Unterschiede zeigen sich hinsichtlich der Teildisziplinen. Trotz der teilweise erheblich divergenten Perspektiven in den jeweiligen Spezialisierungen des Fachs können sich alle Politikwissenschaftler:innen also weitgehend auf eine Positionierung der Parteien einigen. Nennenswerte Unterschiede finden sich bei CDU und CSU, die von Forscher:innen aus dem Bereich der Gender Studies etwas rechter eingeordnet werden. Möglicherweise gewichten die Expert:innen aus dem Teilgebiet der Gender Studies Gleichstellungsthemen etwas stärker und verorten damit die CDU und CSU weiter rechts als ihre Kolleg:innen aus anderen Teildisziplinen. Angesichts der Tatsache, dass die Positionen von acht Parteien über mehr als 10 Teilgebiete hinweg verglichen werden, können diese Unterschiede freilich auch rein zufällig entstanden sein. Ähnliches gilt vermutlich für die wenigen signifikanten, je- 


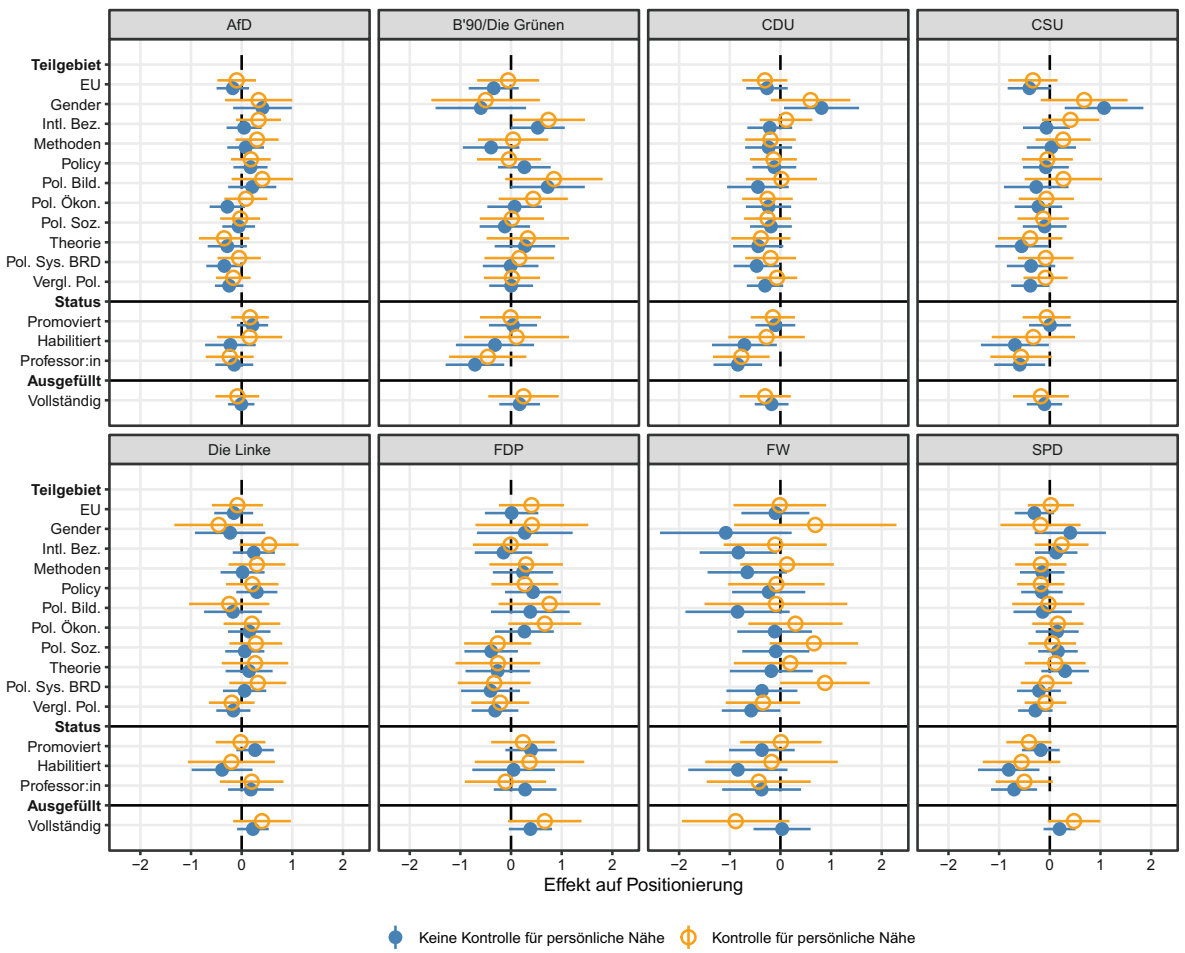

Abb. 5 Effekte des Forschungsschwerpunkts und des akademischen Status auf allgemeine Links-RechtsPositionierung der einzelnen Parteien

doch nicht systematisch erscheinenden Unterschiede bei den Freien Wählern. Aber auch für diese Ergebnisse gilt, dass die Unterschiede verschwinden bzw. deutlich schwächer sind, wenn man für die politische Nähe der Expert:innen kontrolliert.

Abschließend zeigen sich auch keine Unterschiede zwischen Expert:innen, die den OES21 vollständig beantwortet haben und jenen, die nur einen Teil der Fragen beantworteten. Dies spricht dafür, dass der Einschluss der unvollständigen Beobachtungen gerechtfertigt ist.

Insgesamt zeigen die Ergebnisse, dass eine hohe Konsistenz in der Positionierung über Teilgebiete und akademischen Status herrscht. Diese Ergebnisse sind nicht nur für die Positionierungen des OES21 von Relevanz, sie können auch die Planung und Durchführung anderer Expert-Surveys informieren. Auch wenn es sinnvoll erscheint, Expert:innen zu fragen, die direkt zu Parteien und deren Positionierungen forschen, erscheint der offene und breitere Ansatz des OES21 zu mindestens vergleichbaren Ergebnissen zu führen. Wohlgemerkt gilt diese Einschätzung nur für die hier analysierte Links-Rechts-Dimension und nicht zwingend für alle abgefragten Items. Bei spezifischeren Themen wird vermutlich eine größere Expertise notwendig, weshalb eine breite Expert:innen-Befragung an ihre Grenzen stoßen kann. Auch könnte eine engere Auswahl von Expert:innen die Reliabilität gegenüber einer breiten Expert:innenbefragung erhöhen. 


\section{Dimensionalität des Politikraumes}

Die Beschreibung der Parteipositionen zeigt, dass sich die Positionen der Parteien in den verschiedenen Politikfeldern teils stärker, teils weniger stark unterscheiden. Es stellt sich somit die Frage, inwieweit sich der mehrdimensionale Issue-Raum auf einen Politikraum mit einer geringeren Anzahl sogenannter latenter Konfliktdimensionen reduzieren lässt. Dies wäre der Fall, wenn sich die Positionen der Parteien zu konkreten Streitfragen maßgeblich durch ihre Ordnung auf einer grundlegenderen, allgemeinen Konfliktdimension bestimmen lassen. In der Literatur zu westeuropäischen Parteiensystemen wird zumeist von einem zweidimensionalen Politikraum ausgegangen: einer sozio-ökonomischen Konfliktdimension, die das Spannungsfeld zwischen einem starken Wohlfahrststaat und einer liberalen Marktwirtschaft umfasst, sowie einer gesellschaftspolitischen Konfliktdimension, auf der sich säkularliberale und religös-autoritäre Idealvorstellungen gegenüberstehen (Lipset und Rokkan 1967; Pickel 2009).Neuere empirische Literatur legt nahe, dass sich der Inhalt der zweiten, gesellschaftspolitischen Konfliktdimension besser durch einen kulturellen Konflikt aus Gewinnern und Verlierern der Globalisierung definieren lässt (Kriesi et al. 2006), gleichwohl bleibt der Zielkonflikt zwischen einer liberalen und einer autoritären Weltansicht ähnlich.

Um die Dimensionalität der Politikraums zur Bundestagswahl 2021 zu bestimmen, ziehen wir nun alle 22 verfügbaren Positionissues des OES21 heran. Als Kriterium zur Bestimmung der Anzahl der latenten Dimensionen verlassen wir uns auf das Eigenwertkriterium: Es werden so viele Dimensionen zur Beschreibung der Datenmatrix benötigt, wie es Eigenwerte größer eins gibt. Dies führt zu einer dreidimensionalen Lösung unserer Datenmatrix. Es werden also im Folgenden die Ergebnisse einer konfirmatorischen Faktorenanalyse mit drei Dimensionen und Varimax-Rotation vorgestellt (siehe Tab. 1).

Wie erwartet lässt sich ein Faktor als gesellschaftspolitische Konfliktdimension beschreiben und ein weiterer Faktor als sozio-ökonomische Konfliktdimension. Auf dem gesellschaftspolitischen Konflikt laden die Positionsfragen zu Gesellschaftspolitik, Recht und Ordnung, Zuwanderung, Integration, Gleichstellung aller Familien und traditionellen Geschlechterrollenbildern hoch $(>0,7)$. Auch weitere Items zu moralischen und kulturellen Themenfeldern, Leistungen für Migrant:innen, Integration und inklusiver Sprache laden moderat $(>0,5)$.

Auf dem zweiten Faktor laden insbesondere die Positionsfragen zur Wirtschaftspolitik, alle drei Items zur Bildung sowie das Item zum Gender Pay Gap sehr hoch $(>0,7)$. Der dritte Faktor ist ein alleiniger Faktor der Coronapolitik. Nur die beiden Corona-Items laden hier über 0,7. Dies verdeutlicht, dass die Parteipositionen zur Coronapolitik eine neue Konfliktlinie im Politikraum aufspannen, auf der sich die Parteien anders einordnen als auf den beiden etablierten Konfliktdimensionen.

Einige der Items laden auf keinem der drei Faktoren hoch. Dies betrifft zum Beispiel die Klimapolitik. Dieses Item lädt auf allen drei Faktoren moderat, mit Werten um 0,5. Das kann bedeuten, dass die Klimapolitik eine weitere latente Konfliktdimension aufspannt. Da hier jedoch nur ein einzelnes Item abgefragt wurde, kann dies nicht quantitativ überprüft werden. Es kann jedoch festgestellt werden, dass die Parteipositionen zum Konflikt zwischen Klimaschutz und wirtschaftlichem Wachstum 
Tab. 1 Faktorladungen einer konfirmatorischen Faktoranalyse der Positionsschätzungen der Befragten (Varimax-Rotation)

\begin{tabular}{|c|c|c|c|}
\hline & 1. Faktor & 2. Faktor & 3. Faktor \\
\hline & $\begin{array}{l}\text { Gesellschaftspol. } \\
\text { Konfliktdim. }\end{array}$ & $\begin{array}{l}\text { Sozio-ökonom. } \\
\text { Konfliktdim. }\end{array}$ & $\begin{array}{r}\text { Corona- } \\
\text { politik }\end{array}$ \\
\hline $\begin{array}{l}\text { Gleichstellung aller } \\
\text { Familien }\end{array}$ & $\mathbf{0 , 8 3}$ & $-0,41$ & $-0,03$ \\
\hline Feministische Politik & $-0,29$ & 0,19 & 0,28 \\
\hline Coronapolitik Freiheit & 0,00 & 0,24 & $\mathbf{0 , 8 7}$ \\
\hline $\begin{array}{l}\text { Coronapolitik Zentra- } \\
\text { lisierung }\end{array}$ & 0,05 & 0,37 & $\mathbf{0 , 8 2}$ \\
\hline Gesellschaftspolitik & $-0,85$ & 0,22 & 0,04 \\
\hline Recht und Ordnung & $-0,85$ & 0,21 & $-0,02$ \\
\hline Zuwanderung & $-0,79$ & 0,41 & 0,32 \\
\hline $\begin{array}{l}\text { Leistungen für Mi- } \\
\text { grant:innen }\end{array}$ & $\mathbf{0 , 6 0}$ & $-0,48$ & $-0,28$ \\
\hline Integration & 0,69 & $-0,25$ & $-0,19$ \\
\hline Klimapolitik & $-0,48$ & $\mathbf{0 , 5 3}$ & $\mathbf{0 , 5 3}$ \\
\hline Wirtschaftspolitik & $-0,30$ & 0,72 & 0,38 \\
\hline Gemeinschaftsschule & $-0,52$ & 0,76 & 0,19 \\
\hline Frühkindliche Bildung & $-0,31$ & 0,75 & 0,35 \\
\hline Studiengebühren & $-0,25$ & $\mathbf{0 , 8 2}$ & 0,37 \\
\hline $\begin{array}{l}\text { EU legislative Kompe- } \\
\text { tenz }\end{array}$ & $-0,51$ & 0,17 & 0,49 \\
\hline $\begin{array}{l}\text { EU Ausgaben f. eige- } \\
\text { nes Land }\end{array}$ & 0,41 & $-0,31$ & $-0,45$ \\
\hline $\begin{array}{l}\text { EU Reduzierung Un- } \\
\text { gleichheit }\end{array}$ & 0,49 & $-0,70$ & $-0,31$ \\
\hline EU Hilfeleistungen & 0,35 & $-0,54$ & $-0,60$ \\
\hline $\begin{array}{l}\text { EU gemeinsame } \\
\text { Schulden }\end{array}$ & 0,44 & $-0,73$ & $-0,39$ \\
\hline Gender Pay Gap & 0,48 & $-0,71$ & $-0,46$ \\
\hline Geschlechterrollenbilder & $\mathbf{0 , 8 0}$ & $-0,44$ & $-0,22$ \\
\hline Inklusive Sprache & 0,64 & $-0,62$ & $-0,27$ \\
\hline
\end{tabular}

nicht ausschöpfend durch deren Position auf dem latenten sozio-ökonomischen Konflikt beschrieben werden können. ${ }^{6}$ Ähnliches gilt für die Fragen zur Europäischen Integration. Hier stehen jedoch genügend Items zur Verfügung, um die Existenz einer separaten latenten Dimension auszuschließen. Es gibt keinen Faktor, auf welchem alle EU-Items hoch laden. Stattdessen beobachten wir eine gewisse Heterogenität: Während die Frage zur Ausweitung der legislativen Kompetenzen moderat auf dem gesellschaftspolitischen Faktor lädt $(-0,5)$, lädt die Frage zur Reduzierung der so-

\footnotetext{
${ }^{6}$ Die Kreuzladung des Klimaschutz-Items könnte auch dadurch erklärt werden, dass die Fragestellung nach einem Trade-off zwischen der Priorität von Klimaschutz und Wirtschaftswachstum per Definition eine Vermischung beider latenter Konfliktlinien beinhaltet. Wie bereits erwähnt, sollte über eine trennschärfere Frageformulierung nachgedacht werden.
} 

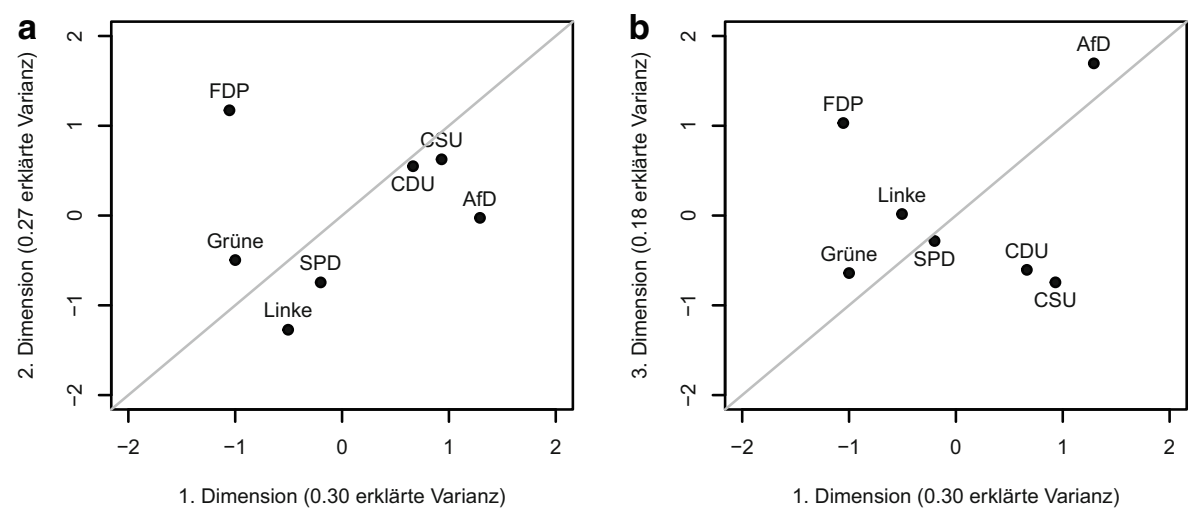

Abb. 6 Parteipositionen nach konfirmatorischer Faktorenanalyse der 22 Items mit 3 latenten Dimensionen

zialen Ungleichheit innerhalb der EU auf dem sozio-ökonomischen Faktor $(-0,7)$. Dies spricht eher dafür, dass die Parteistandpunkte zur Europäischen Integration von verschiedenen kulturellen und sozio-ökonomischen Interessen der Parteien geprägt sind, aber dem Politikfeld keine originäre, eigene latente Dimension zugrunde liegt.

Abb. 6 stellt die Ergebnisse der Faktoranalyse grafisch dar, indem die Mittelwerte der Faktorscores der Parteieinschätzungen als latente Positionen im dreidimensionalen Raum interpretiert werden. Die erste Abbildung bildet die so berechneten Parteipositionen auf der 1. latenten Dimension, also der gesellschaftspolitischen, und der 2. latenten, also der sozio-ökonomischen, Dimension ab. Es ergibt sich das bekannte Bild, in welchem sich alle Parteien nahe der Hauptdiagonalen befinden, mit Ausnahme der FDP, welche gesellschaftspolitisch liberal, aber sozio-ökonomisch rechts angeordnet ist. In der rechten Abbildung sind die Positionen der latenten 3. Dimension und der 1. Dimension dargestellt. Die 3. Dimension beschreibt die Positionen bezüglich der Coronapolitik. Hier zeigt sich eine deutliche Polarisierung zwischen der AfD und der FDP auf der einen Seite, und allen anderen Parteien auf der anderen Seite. AfD und FDP traten bereits vor und während des Bundestagswahlkampfes 2021 als Gegner der Coronapolitik der Regierung auf. Beide lehnten ein starkes Eingreifen des Staates in die Grundrechte der Bürger:innen ab - allerdings mit unterschiedlicher Intensität und aus unterschiedlichen Gründen. Während die FDP ihre Haltung mit den Freiheitsrechten der Bürger:innen und einer stärkeren Eigenverantwortung begründet, nahm die AfD eine populistische Anti-EstablishmentHaltung ein. Inwieweit sich diese Konfliktlinie im deutschen Politikraum behauptet, bleibt jedoch abzuwarten. Es besteht die Hoffnung, dass sich die Pandemiepolitik nicht zum dauerhaften Bestandteil des deutschen Politikraums entwickelt und bereits zur nächsten Bundestagswahl wieder nur zwei latente Dimensionen den politischen Konfliktraum prägen. 


\section{Diskussion}

Der Open Expert Survey 2021 liefert eine umfassende Datenbasis für die Analyse des Parteienwettbewerbs im Kontext der Bundestagswahl 2021. Die erhobenen Daten bilden eine Vielzahl an Themen ab, die in dieser Form in gängigen Expert Surveys nicht enthalten sind. Damit liefert der OES21 die Möglichkeit, den Parteienwettbewerb auch hinsichtlich von Dimensionen zu analysieren, die von internationalen Projekten häufig nicht adressiert werden. Insbesondere seien hier neben den Positionen zur Pandemiepolitik auch die Vielzahl an Positionsfragen zur Bildungspolitik und Themen der Gleichstellung erwähnt. Neben den Positionen der Parteien zu einzelnen Politikfeldern enthält der OES21 auch Angaben über die Wichtigkeit der Themenfelder, welche vertiefte Analysen zu Policy-Salienzen ermöglichen. Daher hoffen wir, dass die produzierten Daten die Grundlage für spannende Forschungsarbeiten sein werden.

Darüber hinaus lässt sich jedoch auch festhalten, dass das Konzept des OES21 vielversprechend ist. Der OES21 kombiniert eine hohe Anzahl an Expert:innen-Einschätzungen mit einer Vielzahl an abgefragten Themen. Wie wir in Bezug auf die Links-Rechts-Positionierung gezeigt haben, führt dieses Konzept nicht zwingend zu einer Verschlechterung der geschätzten Positionen. Im Gegenteil: Die vorgenommenen Positionierungen scheinen über Expert:innen-Gruppen, also über Teildisziplin und akademischen Status, hinweg konsistent zu sein. Daher stellt sich die Frage, ob vergleichbare Studien nicht auch bei anderen Wahlen, insbesondere in anderen Staaten, durchgeführt werden sollten. Auf diesem Weg bestünde dann ein ExpertSurvey-Äquivalent zu Wahlprogrammanalysen, welche ebenfalls zu jeder Wahl neue Positionsdaten produzieren. Die bestehenden, international vergleichenden Expert Surveys hingegen fragen meist zeitgleich in mehreren Ländern die Positionen ab, aber nicht direkt zum Zeitpunkt der jeweiligen Wahl. Wenngleich letzteres Vorgehen auch viele Vorteile bietet, wäre eine Ergänzung durch eine wahlspezifische Positionserhebung wünschenswert. Das Vorgehen des OES21 kann für ein solches Vorhaben eine Grundlage bilden.

Danksagung Wir danken Thilo Dieing, Anusha Fraikin, Isabelle Kohl, Tim Rieth und Torren Frank für hilfreiche Unterstützung. Der OES2021 wurde durch die Projektförderung der DFG (STE 2353/2-1) und des DeZIM unterstützt.

Funding Open Access funding enabled and organized by Projekt DEAL.

Open Access Dieser Artikel wird unter der Creative Commons Namensnennung 4.0 International Lizenz veröffentlicht, welche die Nutzung, Vervielfältigung, Bearbeitung, Verbreitung und Wiedergabe in jeglichem Medium und Format erlaubt, sofern Sie den/die ursprünglichen Autor(en) und die Quelle ordnungsgemäß nennen, einen Link zur Creative Commons Lizenz beifügen und angeben, ob Änderungen vorgenommen wurden.

Die in diesem Artikel enthaltenen Bilder und sonstiges Drittmaterial unterliegen ebenfalls der genannten Creative Commons Lizenz, sofern sich aus der Abbildungslegende nichts anderes ergibt. Sofern das betreffende Material nicht unter der genannten Creative Commons Lizenz steht und die betreffende Handlung nicht nach gesetzlichen Vorschriften erlaubt ist, ist für die oben aufgeführten Weiterverwendungen des Materials die Einwilligung des jeweiligen Rechteinhabers einzuholen. 
Weitere Details zur Lizenz entnehmen Sie bitte der Lizenzinformation auf http://creativecommons.org/ licenses/by/4.0/deed.de.

Interessenkonflikt Keine:r der Autor:innen hat einen Interessenskonflikt (Conflict of Interest) anzuzeigen.

\section{Literatur}

Albright, Jeremy J. 2010. The multidimensional nature of party competition. Party Politics 16(6):699-719. Benoit, Kenneth, und Michael Laver. 2006. Party policy in modern democracies. London: Routledge.

Benoit, Kenneth, Drew Conway, Benjamin E. Lauderdale, Michael Laver, und Slava Mikhaylov. 2016. Crowd-sourced text analysis: Reproducible and agile production of political data. American Political Science Review 110(2):278-295.

Bräuninger, Thomas, Marc Debus, Jochen Müller, und Christian Stecker. 2019a. Die programmatischen Positionen der deutschen Parteien zur Bundestagswahl 2017: Ergebnisse einer Expertenbefragung. In Jahrbuch für Handlungs- und Entscheidungstheorie, Hrsg. Marc Debus, Markus Tepe, und Jan Sauermann, 93-113. Wiesbaden: Springer.

Bräuninger, Thomas, Marc Debus, Jochen Müller, und Christian Stecker. 2019b. Party Competition and Government Formation in Germany: Business as Usual or New Patterns? German Politics 28(1):80-100.

Bräuninger, Thomas, Marc Debus, Jochen Müller, und Christian Stecker. 2020. Parteienwettbewerb in den deutschen Bundesländern. Wiesbaden: Springer.

Dalton, Russell J. 2008. The Quantity and the Quality of Party Systems. Comparative Political Studies 41(7):899-920.

Ganghof, Steffen. 2003. Promises and Pitfalls of Veto Player Analysis. Swiss Political Science Review $9(2): 1-25$.

Ganghof, Steffen. 2006. Strategische Uneinigkeit? Methodische Probleme und normative Implikationen von analytischen Erzählungen über Reformblockaden. In Jahrbuch für Handlungs- und Entscheidungstheorie, Bd. 4, Hrsg. Thomas Bräuninger, Joachim Behnke, 151-177. Wiesbaden: VS.

Golder, Matt, und Jacek Stramski. 2010. Ideological Congruence and Electoral Institutions. American Journal of Political Science 54(1):90-106.

Gross, Martin, und Michael Jankowski. 2020. Lokale Wahlprogramme. Ein blinder Fleck der deutschen Kommunalpolitikforschung? In Neue Koalitionen - alte Probleme: Lokale Entscheidungsprozesse im Wandel, Hrsg. Björn Egner, Detlef Sack, 101-126. Wiesbaden: Springer.

Horn, Alexander. 2019. Can the online crowd match real expert judgments? How task complexity and coder location affect the validity of crowd-coded data. European Journal of Political Research 58(1):236-247.

Kriesi, Hanspeter, Edgar Grande, Romain Lachat, S. Dolezal, S. Bornschier, und T. Frey. 2006. Globalization and the transformation of the national political space: Six European countries compared. European Journal of Political Research 45(6):921-956.

Laver, Michael. 2014. Measuring policy positions in political space. Annual Review of Political Science 17:207-223.

Laver, Michael, und W. Ben Hunt. 1992. Policy and party competition. New York: Routledge.

Laver, Michael, Kenneth Benoit, und John Garry. 2003. Extracting Policy Positions from Political Texts Using Words as Data. American Political Science Review 97(2):311-331.

Lipset, Seymour M., und Stein Rokkan. 1967. Cleavage Structures, Party Systems, and Voter Alignments. An Introduction. In Party Systems, and Voter Alignments: Cross-National Perspectives, Hrsg. Seymour M. Lipset, Stein Rokkan. New York/London: Collier-Macmillan, 1-64.

Müller, Wolfgang C., und Kaare Strøm. 2010. Policy, Office, or Votes? Cambridge: Cambridge University Press.

Pickel, Susanne. 2009. Neue Konflikte - neue gesellschaftliche Koalitionen? Die europäischen Wähler und ihre Parteien - Cleavages in West- und Osteuropa. Universität Greifswald: Habilitationsschrift.

Polk, Jonathan, Jan Rovny, Ryan Bakker, Erica Edwards, Liesbet Hooghe, Seth Jolly, Jelle Koedam, Filip Kostelka, Gary Marks, Gijs Schumacher, Marco Steenbergen, Milada Vachudova, und Marko Zilovic. 2017. Explaining the salience of anti-elitism and reducing political corruption for political parties in Europe with the 2014 Chapel Hill Expert Survey data. Research \& Politics 4(1):1-9.

Saalfeld, Thomas. 2006. Conflict and Consensus in Germany's Bi-cameral System: A Case Study of the Passage of the Agenda 2010. Debatte 14(3):247-269. 
Slapin, Jonathan B., und Sven-Oliver Proksch. 2008. A Scaling Model for Estimating Time-Series Party Positions from Texts. American Journal of Political Science 52(3):705-722.

Volkens, Andrea, Hans-Dieter Klingemann, Ian Budge, Judith Bara, und Michael Dennis McDonald (Hrsg.). 2014. Mapping policy preferences from texts: Statistical solutions for manifesto analysts. Oxford: Oxford University Press.

Zohlnhöfer, Reimut. 1999. Die große Steuerreform 1998/99: Ein Lehrstück für Politikentwicklung bei Parteienwettbewerb im Bundesstaat. Zeitschrift für Parlamentsfragen 30(2):326-345. 